\title{
Variational Iteration Method for the Magnetohydrodynamic Flow over a Nonlinear Stretching Sheet
}

\author{
Lan $X u^{1,2}$ and Eric W. M. Lee ${ }^{3}$ \\ ${ }^{1}$ National Engineering Laboratory for Modern Silk, College of Textile and Engineering, Soochow University, 199 Ren-ai Road, \\ Suzhou, Jiangsu 215123, China \\ ${ }^{2}$ Nantong Textile Institute of Soochow University, 58 Chong-chuan Road, Nantong, Jiangsu 226018, China \\ ${ }^{3}$ Department of Civil and Architectural Engineering, City University of Hong Kong, 83 Tat Chee Avenue, Kowloon Tong, \\ Kowloon, Hong Kong
}

Correspondence should be addressed to Eric W. M. Lee; ericlee@cityu.edu.hk

Received 10 December 2012; Accepted 22 February 2013

Academic Editor: de Dai

Copyright (c) 2013 L. Xu and E. W. M. Lee. This is an open access article distributed under the Creative Commons Attribution License, which permits unrestricted use, distribution, and reproduction in any medium, provided the original work is properly cited.

\begin{abstract}
The variational iteration method (VIM) is applied to solve the boundary layer problem of magnetohydrodynamic flow over a nonlinear stretching sheet. The combination of the VIM and the Padé approximants is shown to be a powerful method for solving two-point boundary value problems consisting of systems of nonlinear differential equations. And the comparison of the obtained results with other available results shows that the method is very effective and convenient for solving boundary layer problems.
\end{abstract}

\section{Introduction}

It is well known that most of the phenomena that arise in mathematical physics and engineering fields can be described by partial differential equations. Recent advances of partial differential equations are stimulated by new examples of applications in fluid mechanics, viscoelasticity, mathematical biology, electrochemistry, and physics. There are many traditional and recently developed methods to give numerical and analytical approximate solutions of nonlinear differential equations such as Euler method, Runge-Kutta method, Taylor series method, Adomian decomposition method [1], Variational iteration method [2,3], Hankel-Padé method [4], DTM-Padé method [5], homotopy perturbation method [6], and Hamiltonian method [7].

In this paper, we consider the model proposed by authors in [1] describing the problem of the boundary layer flow of an incompressible viscous fluid over a nonlinear stretching sheet. The boundary layer flow is often encountered in many engineering and industrial processes. Such processes include the aerodynamic extrusion of plastic sheets, hot rolling, glass fiber production, and so on $[1,4,5]$. And various aspects of the stretching flow problem were discussed by various investigators. Chiam [8] analyzed the MHD flow of a viscous fluid bounded by a stretching surface with power law velocity. He presented the numerical solution of the boundary value problem by utilizing the Runge-Kutta shooting algorithm with Newton iteration. Here, we aim to solve the MHD flow caused by a sheet with nonlinear stretching. The approximate solution of the nonlinear problem is obtained by the variational iteration method.

The variational iteration method [2] is a type of Lagrange multiplier method to find analytical solutions. The method gives the possibility to solve many kinds of non linear equations. In this method, general Lagrange multipliers are introduced to construct correction functional for the problems. The multipliers can be identified optimally via variational theory. It has been used to solve effectively, easily, and accurately a large class of nonlinear problems with approximation [9].

\section{Basic Idea of the VIM}

The basic idea was systematically illustrated and discussed in $[9,10]$. To illustrate the basic idea of the VIM, we consider the 
following general nonlinear system:

$$
L[u(t)]+N[u(t)]=g(t),
$$

where $L, N$, and $g(t)$ are the linear operator, the nonlinear operator, and a given continuous function, respectively. The basic character of the method is to construct a correction functional for the system, which reads

$$
u_{n+1}(t)=u_{n}(t)+\int_{0}^{t} \lambda(s)\left[L u_{n}(s)+N \widetilde{u}_{n}(s)-g(s)\right] d s,
$$

where $\lambda$ is a Lagrange multiplier which can be identified optimally via the variational theory. The subscript $n$ indicates the $n$th approximation, and $\tilde{u}_{n}$ denotes a restricted variation, that is, $\delta \widetilde{u}_{n}=0$.

\section{Problem Statement and Governing Equations}

We consider the magnetohydrodynamic (MHD) flow of an incompressible viscous fluid over a stretching sheet at $y=$ 0 . The fluid is electrically conducting under the influence of an applied magnetic field $B(x)$ normal to the stretching sheet. The induced magnetic field is neglected. The resulting boundary layer equations are as follows [1]:

$$
\begin{gathered}
\frac{\partial u}{\partial x}+\frac{\partial v}{\partial y}=0 \\
u \frac{\partial u}{\partial x}+v \frac{\partial u}{\partial y}=v \frac{\partial^{2} u}{\partial y^{2}}-\frac{\sigma B^{2}(x)}{\rho} u
\end{gathered}
$$

where $u$ and $v$ are the velocity components in the $x$ and $y$ directions, respectively, $\nu$ is the kinematic viscosity, $\rho$ is the fluid density, and $\sigma$ is the electrical conductivity of the fluid. In (4), the external electric field and the polarization effects are negligible, and in [8]

$$
B(x)=B_{0} x^{(n-1) / 2} .
$$

The boundary conditions corresponding to the nonlinear stretching of a sheet are

$$
\begin{gathered}
u(x, 0)=c x^{n}, \quad v(x, 0)=0, \\
u(x, y) \longrightarrow 0 \quad \text { as } y \longrightarrow \infty .
\end{gathered}
$$

Upon making use of the following substitutions:

$$
\begin{gathered}
\eta=\sqrt{\frac{c(n+1)}{2 \nu}} x^{(n-1) / 2} y, \quad u=c x^{n} f^{\prime}(\eta), \\
v=-\sqrt{\frac{c \nu(n+1)}{2}} x^{(n-1) / 2}\left[f(\eta)+\frac{n-1}{n+1} \eta f^{\prime}(\eta)\right],
\end{gathered}
$$

Substituting (8) into (3)-(6), the resulting nonlinear differential system can be written in the following form:

$$
\begin{gathered}
f^{\prime \prime \prime}+f f^{\prime \prime}-\beta f^{\prime 2}-M f^{\prime}=0, \\
f(0)=0, \quad f^{\prime}(0)=1, \quad f^{\prime}(\infty)=0,
\end{gathered}
$$

where

$$
\beta=\frac{2 n}{1+n}, \quad M=\frac{2 \sigma B_{0}^{2}}{\rho c(1+n)} .
$$

The parameter $\beta$ is a measure of the pressure gradient, and $M$ is the magnetic parameter. Positive $\beta$ denotes the favorable negative pressure gradient, and negative $\beta$ denotes the unfavorable positive pressure gradient; naturally, $\beta=0$ denotes the flat plate. For the special case of $\beta=1$, the exact analytical solution of (9) is [11]

$$
f(\eta)=\frac{1-\exp (-\sqrt{1+M} \eta)}{\sqrt{1+M}} .
$$

\section{Approximate Solution by the VIM}

In order to obtain VIM solution of (9), we construct a correction functional which reads

$$
\begin{aligned}
f_{n+1}(\eta) & \\
=f_{n}(\eta)+\int_{0}^{\eta} \lambda(\tau) & {\left[\frac{\partial^{3} f_{n}(\tau)}{\partial \tau^{3}}+\widetilde{f}_{n}(\tau) \frac{\partial^{2} \widetilde{f}_{n}(\tau)}{\partial \tau^{2}}-\beta\right.} \\
& \left.\times\left(\frac{\partial \tilde{f}_{n}(\tau)}{\partial \tau}\right)^{2}-M \frac{\partial \tilde{f}_{n}(\tau)}{\partial \tau}\right] d \tau,
\end{aligned}
$$

where $\lambda(\tau)$ is the general Lagrangian multiplier which can be identified optimally via the variational theory. And $\tilde{f}_{n}(\tau)$ is considered as a restricted variation, that is, $\delta \tilde{f}_{n}(\tau)=0$. We omit asterisks for simplicity. Its stationary conditions can be obtained as follows:

$$
1+\left.\lambda^{\prime \prime}(\tau)\right|_{\tau=\eta}=0,\left.\quad \lambda^{\prime}(\tau)\right|_{\tau=\eta}=0, \quad \lambda^{\prime \prime \prime}(\tau)=0 .
$$

The Lagrange multipliers can be readily identified as the following form:

$$
\lambda(\tau)=-\frac{1}{2}(\tau-\eta)^{2} .
$$

As a result, we obtain the following variational iteration formula

$$
\begin{aligned}
f_{n+1}(\eta) & \\
=f_{n}(\eta)-\frac{1}{2} \int_{0}^{\eta}(\tau-\eta)^{2} & {\left[\frac{\partial^{3} f_{n}(\tau)}{\partial \tau^{3}}+\tilde{f}_{n}(\tau) \frac{\partial^{2} \tilde{f}_{n}(\tau)}{\partial \tau^{2}}-\beta\right.} \\
& \left.\times\left(\frac{\partial \tilde{f}_{n}(\tau)}{\partial \tau}\right)^{2}-M \frac{\partial \tilde{f}_{n}(\tau)}{\partial \tau}\right] d \tau .
\end{aligned}
$$

Now, we assume that an initial approximation

$$
f_{0}(\eta)=a+b \eta+c \eta^{2}
$$


where $a, b$, and $c$ are unknown constants to be further determined.

By the iteration formula (16) and the initial approximation (17), we can obtain directly the first-order approximate solution as follows:

$$
\begin{aligned}
f_{1}(\eta)=f_{0}(\eta)-\frac{1}{2} \int_{0}^{\eta}(\tau-\eta)^{2} & \times\left[\frac{\partial^{3} f_{0}(\tau)}{\partial \tau^{3}}+f_{0}(\tau) \frac{\partial^{2} f_{0}(\tau)}{\partial \tau^{2}}\right. \\
& \left.-\beta\left(\frac{\partial f_{0}(\tau)}{\partial \tau}\right)^{2}-M \frac{\partial f_{0}(\tau)}{\partial \tau}\right] d \tau \\
= & a+b \eta+c \eta^{2}-\frac{c^{2}}{30} \eta^{5}+\frac{b M}{6} \eta^{3}+\frac{c M}{12} \eta^{4} \eta^{5} \\
& +\frac{\beta b^{2}}{6} \eta^{3}+\frac{\beta c^{2}}{15}-\frac{a c}{3} \eta^{3}-\frac{b c}{12} \eta^{4}+\frac{b c \beta}{6} \eta^{4} \\
= & a+b \eta+c \eta^{2}+\frac{b M+\beta b^{2}-2 a c}{6} \eta^{3} \\
& +\frac{c M+b c(2 \beta-1)}{12} \eta^{4}+\frac{(2 \beta-1) c^{2}}{30} \eta^{5}
\end{aligned}
$$

Making use of the initial conditions $f(0)=0, f^{\prime}(0)=1$, we can readily obtain the results as follows:

$$
a=0, \quad b=1, \quad c=\frac{1}{2} f^{\prime \prime}(0),
$$

where $f^{\prime \prime}(0)=\alpha$ will be examined in this work, according the initial condition $f^{\prime}(\infty)=0$.

Then,

$$
\begin{aligned}
f_{1}(\eta)= & \eta+\frac{1}{2} \alpha \eta^{2}+\frac{M+\beta}{6} \eta^{3}+\frac{\alpha(M+2 \beta-1)}{24} \eta^{4} \\
& +\frac{(2 \beta-1) \alpha^{2}}{120} \eta^{5} .
\end{aligned}
$$

And the following second-order approximate solution can be obtained

$$
\begin{aligned}
& f_{2}(\eta)=f_{1}(\eta)-\frac{1}{2} \int_{0}^{\eta}(\tau-\eta)^{2} \\
& \times {\left[\frac{\partial^{3} f_{1}(\tau)}{\partial \tau^{3}}+f_{1}(\tau) \frac{\partial^{2} f_{1}(\tau)}{\partial \tau^{2}}\right.} \\
&\left.-\beta\left(\frac{\partial f_{1}(\tau)}{\partial \tau}\right)^{2}-M \frac{\partial f_{1}(\tau)}{\partial \tau}\right] d \tau \\
&=\eta+\frac{1}{2} \alpha \eta^{2}+\frac{M+\beta}{6} \eta^{3}+\frac{\alpha(M+2 \beta-1)}{24} \eta^{4}
\end{aligned}
$$

$$
\begin{aligned}
& +\left[\frac{(2 \beta-1) \alpha^{2}}{120}+\frac{\beta^{2}}{60}+\frac{\beta M}{40}-\frac{1}{60}+\frac{M^{2}}{120}-\frac{M}{60}\right] \eta^{5} \\
& +\left(\frac{\beta^{2} \alpha}{72}+\frac{\beta M \alpha}{72}-\frac{\beta \alpha}{60}+\frac{M^{2} \alpha}{720}-\frac{M \alpha}{90}+\frac{\alpha}{240}\right) \eta^{6} \\
& +\left(\frac{\beta^{3}}{840}+\frac{\beta^{2} M}{420}+\frac{\beta^{2} \alpha^{2}}{252}-\frac{\beta^{2}}{1260}+\frac{\beta M^{2}}{840}+\frac{\beta M \alpha^{2}}{504}\right. \\
& \left.-\frac{\beta M}{630}-\frac{2 \beta \alpha^{2}}{315}-\frac{M^{2}}{1260}-\frac{M \alpha^{2}}{630}+\frac{11 \alpha^{2}}{5040}\right) \eta^{7} \\
& +\left(\frac{\beta^{3} \alpha}{1008}+\frac{\beta^{2} M \alpha}{672}+\frac{\beta^{2} \alpha^{3}}{2016}-\frac{5 \beta^{2} \alpha}{4032}+\frac{\beta M^{2} \alpha}{2016}\right. \\
& -\frac{13 \beta M \alpha}{8064}-\frac{7 \beta \alpha^{3}}{1260}+\frac{\beta \alpha}{2688}-\frac{M^{2} \alpha}{2688}+\frac{M \alpha}{2688} \\
& \left.+\frac{11 \alpha^{3}}{40320}\right) \eta^{8} \\
& +\left(\frac{\beta^{3} \alpha^{2}}{2592}+\frac{\beta^{2} M \alpha^{2}}{2592}-\frac{37 \beta^{2} \alpha^{2}}{60480}+\frac{\beta M^{2} \alpha^{2}}{18144}\right. \\
& -\frac{13 \beta M \alpha^{2}}{25920}+\frac{53 \beta \alpha^{2}}{181440}-\frac{M^{2} \alpha^{2}}{24192}+\frac{M \alpha^{2}}{6480} \\
& \left.-\frac{\alpha^{2}}{24192}\right) \eta^{9} \\
& +\left(\frac{\beta^{3} \alpha^{3}}{12960}+\frac{\beta^{2} M \alpha^{3}}{25920}-\frac{\beta^{2} \alpha^{3}}{7200}-\frac{13 \beta M \alpha^{3}}{259200}\right. \\
& \left.+\frac{7 \beta \alpha^{3}}{86400}+\frac{M \alpha^{3}}{64800}-\frac{\alpha^{3}}{64800}\right) \eta^{10} \\
& +\left(\frac{\beta^{3} \alpha^{4}}{142560}-\frac{\beta^{2} \alpha^{4}}{79200}+\frac{7 \beta \alpha^{4}}{950400}-\frac{\alpha^{4}}{712800}\right) \eta^{11} .
\end{aligned}
$$

Therefore, according to (13), we can easily obtain higherorder approximate solution as follows:

$$
f(\eta)=r_{0}+r_{1} \eta+r_{2} \eta^{2}+r_{3} \eta^{3}+r_{4} \eta^{4}+r_{5} \eta^{5}+\cdots,
$$

by using mathematical software such as MATLAB.

It is evident that the main problem for solving (21) is to obtain the value of $f^{\prime \prime}(0)$, then we can resort to any numerical integration routine to obtain the solution of the problem. For this purpose, we will employ the Padé method to determine this unknown value with high accuracy.

\section{Padé Approximation}

It is well known that Padé approximations [12] have the advantage of manipulating the polynomial approximation into a rational function of polynomials. This manipulation provides us with more information about the mathematical behavior of the solution. Besides that, power series are not 
TABLE 1: Comparison of the values of $f^{\prime \prime}(0)$ obtained by the variational iteration method and other methods [1] for various values of $M$ when $\beta=1$.

\begin{tabular}{lccc}
\hline$M$ & VIM & ADM [1] & Exact [1] \\
\hline 1.0 & -1.41421 & -1.41421 & -1.41421 \\
5.0 & -2.44948 & -2.44948 & -2.44948 \\
10.0 & -3.31662 & -3.31662 & -3.31662 \\
50.0 & -7.14142 & -7.14142 & -7.14142 \\
100.0 & -10.04987 & -10.04987 & -10.04987 \\
500.0 & -22.38302 & -22.38302 & -22.38302 \\
\hline
\end{tabular}

TABLE 2: Comparison of the values of $f^{\prime \prime}(0)$ obtained by the variational iteration method and the modified Adomian decomposition method [1] for various values of $\beta$ and $M$.

\begin{tabular}{lcccccc}
\hline \multirow{2}{*}{$M$} & \multicolumn{2}{c}{$\beta=-1.5$} & \multicolumn{2}{c}{$\beta=1.5$} & \multicolumn{2}{c}{$\beta=5$} \\
& VIM & ADM [1] & VIM & ADM [1] & VIM & ADM [1] \\
\hline 1.0 & -0.6530 & -0.6532 & -1.5253 & -1.5252 & -2.1529 & -2.1528 \\
5.0 & -2.0852 & -2.0852 & -2.5162 & -2.5161 & -2.9414 & -2.9414 \\
10 & -3.0562 & -3.0562 & -3.3663 & -3.3663 & -3.6957 & -3.6956 \\
50 & -7.0239 & -7.0239 & -7.1647 & -7.1647 & -7.3256 & -7.3256 \\
100 & -9.9667 & -9.9666 & -10.0776 & -10.0776 & -10.1816 & -10.1816 \\
500 & -22.3458 & -22.3457 & -22.3905 & -22.3904 & -22.4426 & -22.4425 \\
\hline
\end{tabular}

useful for large values of $\eta$, say $\eta=\infty$. This can be attributed to the possibility that the radius of convergence may not be sufficiently large to contain the boundaries of the domain. Therefore, the combination of the series solution through the decomposition method or any other series solution method with the Padé approximation provides an effective tool for handling boundary value problems on infinite or semi-infinite domains. Furthermore, it is noted that Padé approximants can be easily evaluated by using Matlab.

Therefore, we suppose that the solution $f(\eta)$ can be expanded as a Taylor series about $\eta=0$

$$
f(\eta)=\sum_{j=0}^{\infty} f_{j} \eta^{j}
$$

Padé approximant, symbolized by $[S / N]$, is a rational function defined by

$$
\left[\frac{S}{N}\right](\eta)=\frac{\sum_{j=0}^{S} p_{j} \eta^{j}}{\sum_{j=0}^{N} q_{j} \eta^{j}} .
$$

If we selected $S=N$, then the approximants $[N / N]$ are called diagonal approximants. More importantly, the diagonal approximants are the most accurate approximants; therefore, we have to construct only diagonal approximants.

Then,

$$
\begin{aligned}
& \frac{p_{0}+p_{1} \eta+p_{2} \eta^{2}+p_{3} \eta^{3}+\cdots+p_{N} \eta^{N}}{q_{0}+q_{1} \eta+q_{2} \eta^{1}+q_{3} \eta^{3}+\cdots+q_{N} \eta^{N}} \\
& =r_{0}+r_{1} \eta+r_{2} \eta^{2}+r_{3} \eta^{3}+r_{4} \eta^{4}+\cdots
\end{aligned}
$$

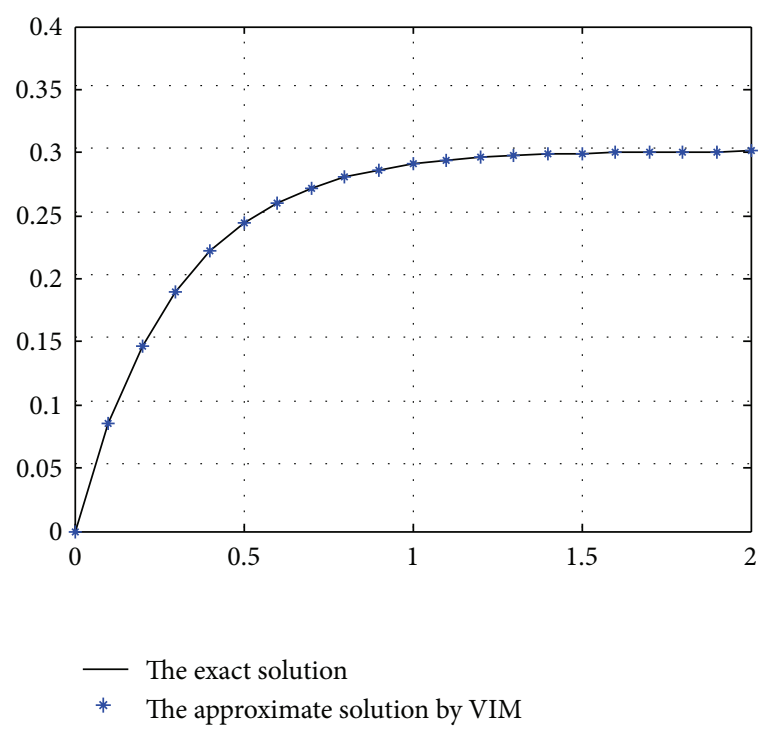

FIgURE 1: Comparison between the approximate solution by the VIM and exact solution for $\beta=1$ and $M=10$.

By using cross multiplication in (25), we find

$$
\begin{aligned}
p_{0}+ & p_{1} \eta+p_{2} \eta^{2}+p_{3} \eta^{3}+\cdots+p_{N} \eta^{N} \\
= & r_{0} q_{0}+\left(r_{1} q_{0}+q_{1} r_{0}\right) \eta+\left(r_{2} q_{0}+q_{1} r_{1}+q_{2} r_{0}\right) \eta^{2} \\
& +\left(r_{3} q_{0}+q_{1} r_{2}+q_{2} r_{1}+q_{3} r_{0}\right) \eta^{3}+\cdots .
\end{aligned}
$$

Using the boundary condition $f^{\prime}(\infty)=0$, the diagonal approximant $[N / N]$ vanishes if the coefficient of $\eta$ with the highest power in the numerator vanishes. By putting the coefficients of the highest power of $\eta$ equal to zero, we can easily obtain the values of $f^{\prime \prime}(0)$ listed in Tables 1 and 2 and Figure 1, using Matlab. The order of Padé approximation [12/12] has sufficient accuracy; on the other hand, if the order of Padé approximation increases, the accuracy of the solution increases.

Substituting (21) and the value of $f^{\prime \prime}(0)$ into (8), we can easily obtain the second-order approximate solution of (3)(4).

\section{Conclusion}

In this paper, the variational iteration method is used to obtain approximate solutions of magnetohydrodynamics boundary layer equations. The analytical solutions of the governing nonlinear boundary layer problem are obtained. Without using the Padé approximation, the analytical solution that were obtained by the VIM cannot satisfy the boundary condition at infinity $f^{\prime}(\infty)=0$. The combination of the VIM and the Padé approximants is shown to be a powerful method for solving two-point boundary value problems consisting of systems of nonlinear differential equations. And the obtained solutions are in good agreement with exact values. 


\section{Acknowledgments}

The work described in this paper was fully supported by a grant from the Research Grants Council of the Hong Kong Special Administrative Region, China (CityU 116308) and Natural Science Foundation of the Jiangsu Higher Education Institutions of China (Grant no. 12KJB130002).

\section{References}

[1] T. Hayat, Q. Hussain, and T. Javed, "The modified decomposition method and Padé approximants for the MHD flow over a non-linear stretching sheet," Nonlinear Analysis: Real World Applications, vol. 10, no. 2, pp. 966-973, 2009.

[2] J. H. He, "Variational iteration method-a kind of non-linear analytical technique: some examples," International Journal of Non-Linear Mechanics, vol. 34, no. 4, pp. 699-708, 1999.

[3] L. Xu, "Variational iteration method for solving integral equations," Computers \& Mathematics with Applications, vol. 54, no. 7-8, pp. 1071-1078, 2007.

[4] S. Abbasbandy and H. R. Ghehsareh, "Solutions of the magnetohydrodynamic flow over a nonlinear stretching sheet and nano boundary layers over stretching surfaces," International Journal for Numerical Methods in Fluids, vol. 70, pp. 1324-1340, 2012.

[5] M. M. Rashidi, "The modified differential transform method for solving MHD boundary-layer equations," Computer Physics Communications, vol. 180, no. 11, pp. 2210-2217, 2009.

[6] J.-H. He, "Homotopy perturbation method with an auxiliary term," Abstract and Applied Analysis, vol. 2012, Article ID 857612, 7 pages, 2012.

[7] L. Xu and J. H. He, "Determination of limit cycle by Hamiltonian approach for strongly nonlinear oscillators," International Journal of Nonlinear Sciences and Numerical Simulation, vol. 11, no. 12, pp. 1097-1101, 2010.

[8] T. C. Chaim, "Hydromagnetic flow over a surface stretching with a power-law velocity," International Journal of Engineering Science, vol. 33, pp. 429-435, 1995.

[9] J.-H. He and X.-H. Wu, "Variational iteration method: new development and applications," Computers \& Mathematics with Applications, vol. 54, no. 7-8, pp. 881-894, 2007.

[10] J.-H. He, "A short remark on fractional variational iteration method," Physics Letters A, vol. 375, no. 38, pp. 3362-3364, 2011.

[11] K. B. Pavlov, "Magnetohydrodynamic flow of an incompressible viscous fluid caused by deformation of a surface," Magnitnaya Gidrodinamika, vol. 4, pp. 146-147, 1975.

[12] G. A. Baker, Essentials of Padé Approximants, Academic press, London, UK, 1975. 


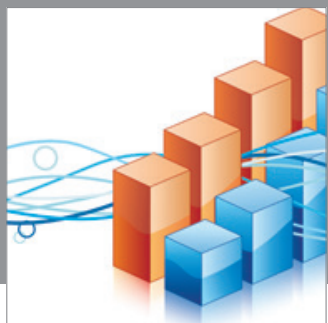

Advances in

Operations Research

mansans

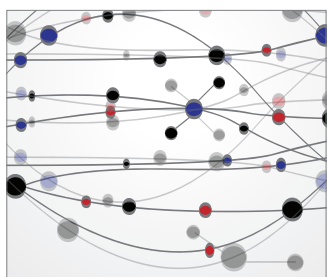

The Scientific World Journal
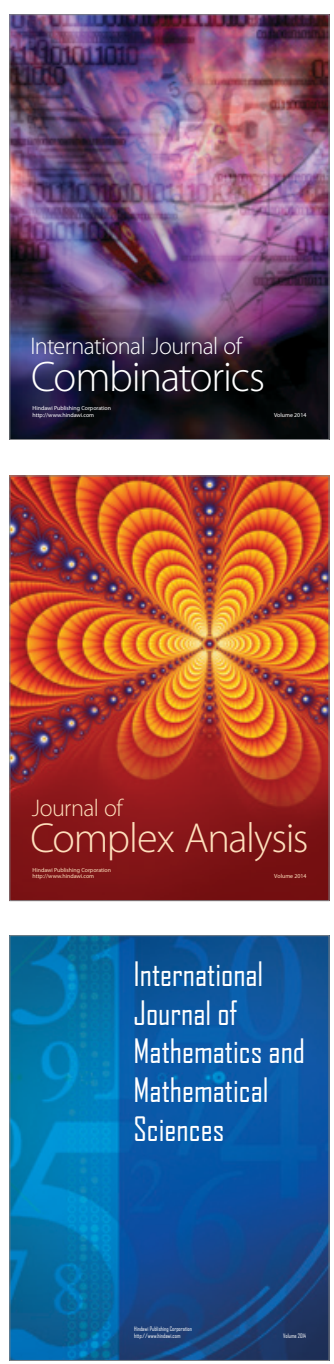
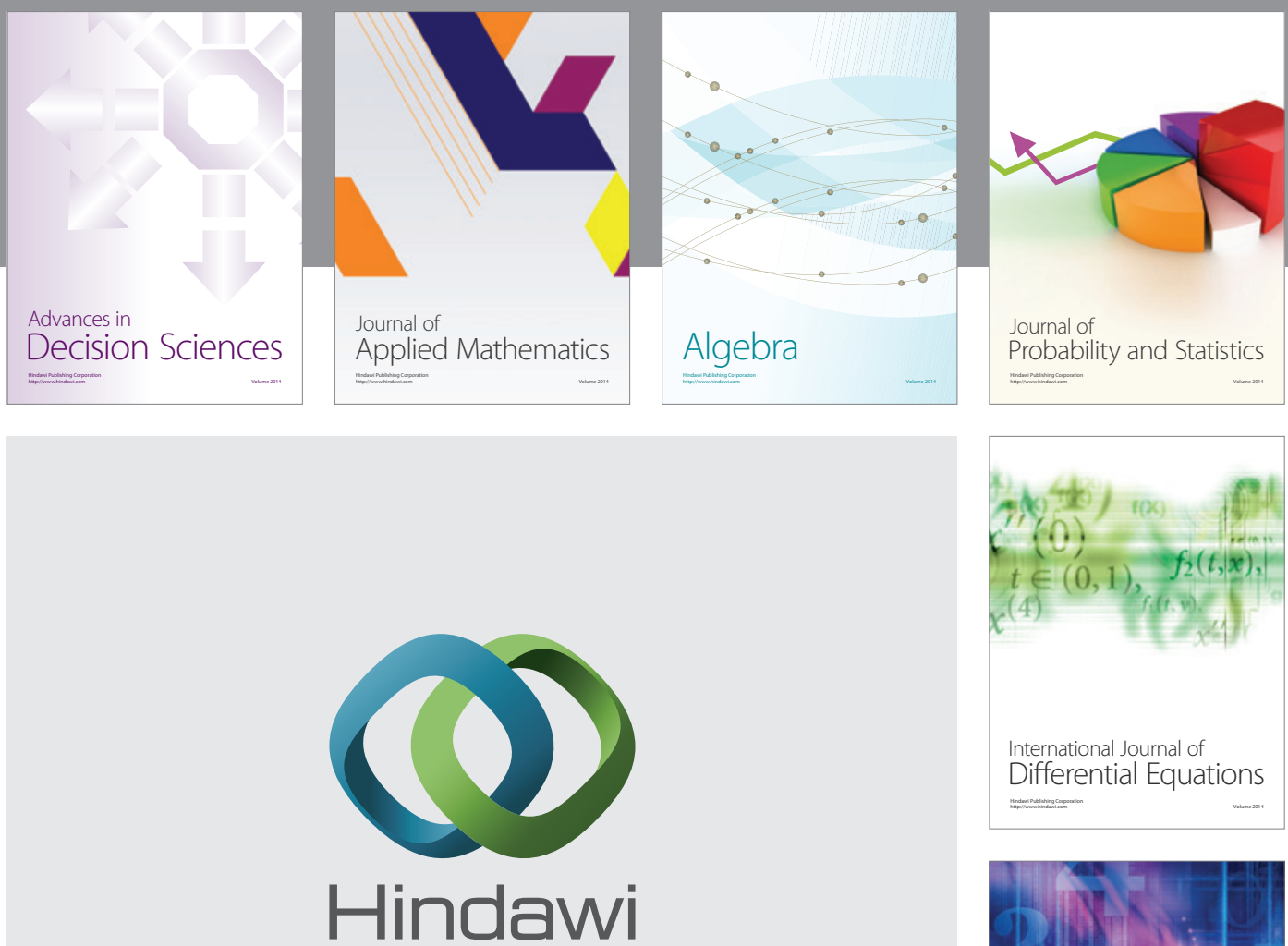

Submit your manuscripts at http://www.hindawi.com
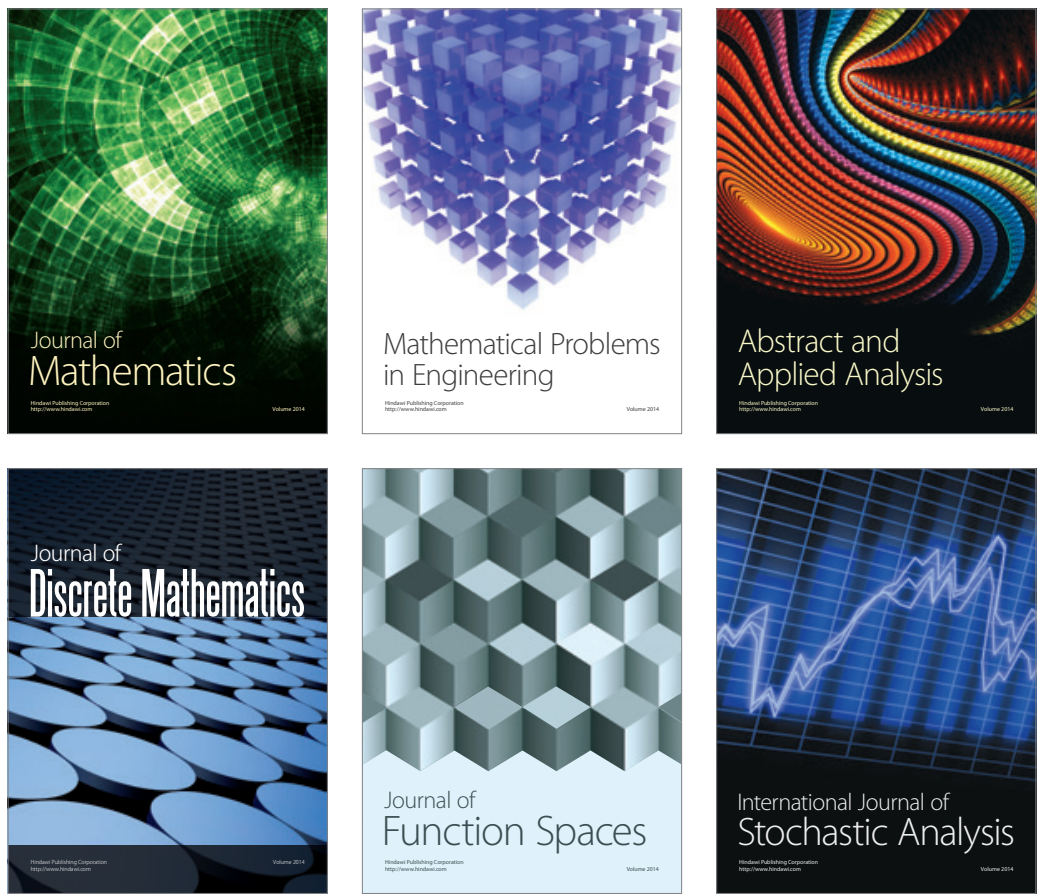

Journal of

Function Spaces

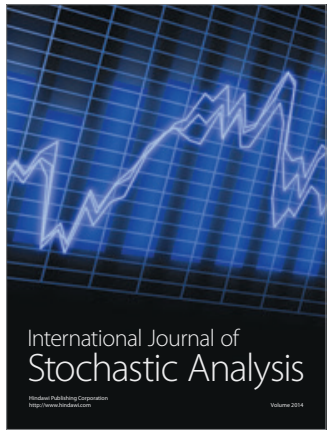

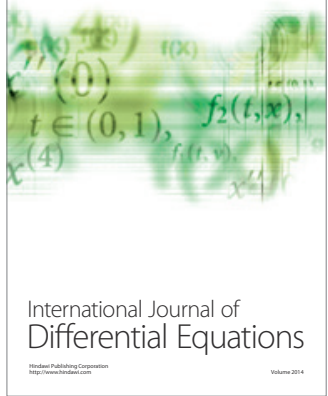
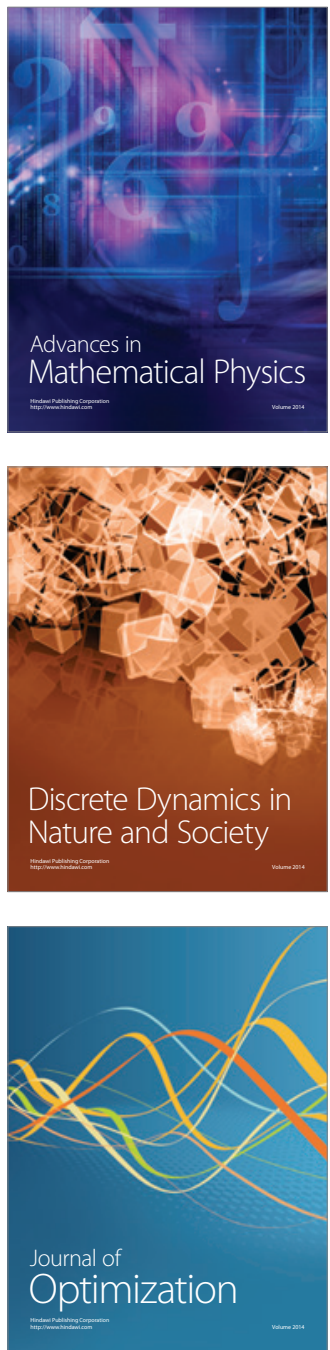